\title{
Characterization of metal contacts for two-dimensional MoS2 nanoflakes
}

Walia, Sumeet; Balendhran, Sivacarendran; Wang, Yichao; Ab Kadir, Rosmalini; Zoolfakar, Ahmad; Atkin, Paul; Ou, Jianzhen

https://researchrepository.rmit.edu.au/esploro/outputs/9921859260701341/filesAndLinks?institution=61RMIT_INST\&index=null

Walia, S., Balendhran, S., Wang, Y., Ab Kadir, R., Zoolfakar, A., Atkin, P., Ou, J., Sriram, S., Kalantar Zadeh, K., \& Bhaskaran, M. (2013). Characterization of metal contacts for two-dimensional MoS2 nanoflakes. Applied Physics Letters, 103(23), 232105-1-232102-232104. https://doi.org/10.1063/1.4840317 Document Version: Accepted Manuscript

Published Version: https://doi.org/10.1063/1.4840317

Repository homepage: https://researchrepository.rmit.edu.au (c) 2013 AIP Publishing LLC

Downloaded On 2023/04/26 23:08:02 +1000 
Thank you for downloading this document from the RMIT Research Repository.

The RMIT Research Repository is an open access database showcasing the research outputs of RMIT University researchers.

RMIT Research Repository: http://researchbank.rmit.edu.au/

\section{Citation:}

Walia, S, Balendhran, S, Wang, Y, Ab Kadir, R, Zoolfakar, A, Atkin, P, Ou, J, Sriram, S, Kalantar Zadeh, K and Bhaskaran, M 2013, 'Characterization of metal contacts for two-dimensional MoS2 nanoflakes', Applied Physics Letters, vol. 103, pp. 232105-1-232102-4.

See this record in the RMIT Research Repository at:

http://researchbank.rmit.edu.au/view/rmit:22637

Version: Accepted Manuscript

Copyright Statement: (c) 2013 AIP Publishing LLC

Link to Published Version:

http://dx.doi.org/10.1063/1.4840317 


\title{
Characterization of metal contacts for two-dimensional $\mathrm{MoS}_{2}$
}

\section{nanoflakes}

Sumeet Walia, ${ }^{1,2, a)}$ Sivacarendran Balendhran, ${ }^{1,2}$ Yichao Wang, ${ }^{2}$ Rosmalini Ab Kadir, ${ }^{2}$ Ahmad Sabirin Zoolfakar, ${ }^{2}$ Paul Atkin, ${ }^{2}$ Jian Zhen Ou, ${ }^{2}$ Sharath Sriram, ${ }^{1,2}$ Kourosh Kalantarzadeh $^{2, a)}$ and Madhu Bhaskaran ${ }^{1,2, a)}$

${ }^{1}$ Functional Materials and Microsystems Research Group, RMIT University, Melbourne, Victoria 3000, Australia

${ }^{2}$ School of Electrical and Computer Engineering, RMIT University, Melbourne, Victoria 3000, Australia

\begin{abstract}
While layered materials are increasingly investigated for their potential in nanoelectronics, their functionality and efficiency depend on charge injection into the materials via metallic contacts. This work explores the characteristics of different metals (aluminium, tungsten, gold and platinum) deposited on to nanostructured thin films made of two-dimensional (2D) $\mathrm{MoS}_{2}$ flakes. Metals are chosen based on their work functions relative to the electron affinity of $\mathrm{MoS}_{2}$. It is observed, and analytically verified, that lower work functions of the contact metals lead to smaller Schottky barrier heights and consequently higher charge carrier injection through the contacts.
\end{abstract}

\section{KEYWORDS}

Contacts, work function, electron affinity, nanoflakes, $\mathrm{MoS}_{2}$ 
Two-dimensional (2D) materials are gaining significant attention due to their unique electrical, mechanical and optical properties, which are different than those of their bulk counterparts. ${ }^{1,2}$ In particular, 2D transition metal dichalcogenides are attracting extensive research interest due to possibilities of electronic band structure manipulations. ${ }^{1,2}$ Amongst them, molybdenum disulphide $\left(\mathrm{MoS}_{2}\right)$ which is a n-type semiconductor, has been the most popular material owing to its relatively small bandgap $(1.2 \mathrm{eV}$ for multi-layer and $1.8 \mathrm{eV}$ for monolayer), modest electron mobilities and transition from indirect to direct bandgap when it is exfoliated to monolayer thickness. ${ }^{1,2}$ Amidst the three known polymorphs of $\mathrm{MoS}_{2}(1 \mathrm{~T}, 2 \mathrm{H}$ and $3 \mathrm{R}), 2 \mathrm{H}-\mathrm{MoS}_{2}$ is the naturally occurring and thermodynamically stable phase. ${ }^{1,2}$ Bulk $2 \mathrm{H}-\mathrm{MoS}_{2}$ is composed of atomically thin planes comprising of covalently bonded S-Mo-S structures across the planes and weak van der Waals forces between the planes. ${ }^{3}$ Such a crystal structure enables the realisation of atomically thin stable 2D sheets of $\mathrm{MoS}_{2}$ via mechanical and liquid exfoliation techniques. ${ }^{1,2}$

2D $\mathrm{MoS}_{2}$ has been applied in the development of a variety of devices including field effect transistors, electrically driven optical devices and solar cells, as well as electrochemical devices. $^{4,5,6}$ All these devices rely on electrical contacts for their operation. As such, understanding metal/2D $\mathrm{MoS}_{2}$ interface is crucial in such devices and the nature of the metal/2D MoS 2 depends on the electronic band structures of both the metal and $2 \mathrm{D} \mathrm{MoS}$.

In this letter, we provide a thorough experimental analysis of metal/nanostructured thin films made of $2 \mathrm{D} \mathrm{MoS}$ nanoflakes contacts for selected metals. The choice of metal contacts is based on their work functions $\left(\Phi_{\mathrm{M}}\right)$ relative to the electron affinity $(\chi)$ of $\operatorname{MoS}_{2}$. Relatively low work function metals, aluminium (Al) and tungsten (W), are studied along with relatively high work function metals, gold $(\mathrm{Au})$ and platinum $(\mathrm{Pt})$, in order to provide a wide-ranging picture of the behaviour of the metal/2D $\mathrm{MoS}_{2}$ contacts. 
In this study, a grinding-sonication liquid exfoliation technique, adapted from the work of Yao et al., ${ }^{7}$ is employed as a high yield method for producing suspensions of 2D $\mathrm{MoS}_{2}$ nanoflakes. ${ }^{8}$ Atomic force microscopy (AFM) was employed to assess the thickness of the $\mathrm{MoS}_{2}$ nanoflakes. It can be seen from Fig. 1(a) that typical $\mathrm{MoS}_{2}$ nanoflakes comprise of 6 to 8 fundamental layers $(1 \text { layer }=0.65 \mathrm{~nm})^{4}$. Measurements using a large number of AFM images shows that the majority of $\mathrm{MoS}_{2}$ nanoflakes exhibit thicknesses in the range of 2-7 monolayers. ${ }^{9}$ Dynamic light scattering (DLS) analysis was conducted to assess the average lateral dimensions of the exfoliated nanoflakes (Fig. 1(b)) to be approximately $100 \mathrm{~nm}$. The nanoflake suspension was drop-casted onto electrically conductive fluorine-doped tin oxide (FTO) substrates and left to dry under ambient conditions, resulting in thin films of $\sim 500 \mathrm{~nm}$ thickness.

Raman spectroscopy was carried out to further characterise the $\mathrm{MoS}_{2}$ thin films and estimate the number of layers they are comprised of. Figure 2(a) shows the Raman spectra of the bulk and the nanostructured $\mathrm{MoS}_{2}$ thin film. The two dominant modes ( $E_{2 \mathrm{~g}}^{1}$ and $A_{1 \mathrm{~g}}$ ) reflect the vibrations of the Mo-S bond along the horizontal and vertical planes, respectively. ${ }^{10}$ For the $\mathrm{MoS}_{2}$ nanoflakes, the $A_{1 \mathrm{~g}}$ mode showed no significant shift, while the $E_{2 \mathrm{~g}}^{1}$ mode was observed to have red shifted in comparison to their bulk counterpart. This indicates a dominant presence of few-layered (<7 layers) $\mathrm{MoS}_{2}$ nanoflakes in the thin film, ${ }^{9,11}$ which is also in agreement to the AFM analysis presented earlier.

X-ray diffraction (XRD) was utilized to determine the crystallinity of the drop-casted 2D $\mathrm{MoS}_{2}$ nanostructured thin films. It can be seen from Fig. 2(b) that the dominant peak reflecting the (002) plane (ICDD Card no: $77-1716$ ) was observed at $14.4^{\circ}$. In contrast to the XRD pattern of the bulk $\mathrm{MoS}_{2}$, the nanostructured $\mathrm{MoS}_{2}$ thin film did not reveal the presence 
of (100), (106), (006), (105) and (008) planes. This reflects the nature of the thin films which consist of 2D MoS${ }_{2}$ nanoflakes composed of mostly (002) planes.

High resolution transmission electron microscopy (HRTEM) was conducted in order to verify the crystal structure of the $2 \mathrm{D} \mathrm{MoS}$ nanoflakes. The HRTEM image of the edges of the nanoflakes (Fig. 2(c)) confirms the presence of 2D structures with only several fundamental layers. The inset of Fig. 2(c) shows that the parallel lattice fringes of the $\mathrm{MoS}_{2}$ nanoflakes are $0.27 \mathrm{~nm}$ apart, which is in agreement with previously reported values for $2 \mathrm{H}$ $\mathrm{MoS}_{2}{ }^{1}$

In order to investigate the properties of metals when contacted with nanostructured

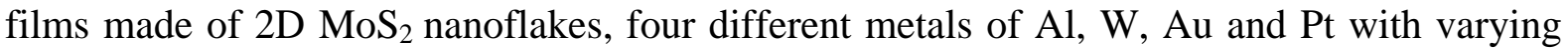
work functions were considered. $\mathrm{Al}\left(\Phi_{\mathrm{Al}}=4.54 \mathrm{eV}\right)^{12}$ and $\mathrm{W}\left(\Phi_{\mathrm{W}}=5.09 \mathrm{eV}\right)^{12}$ were chosen as the low work function metals, while $\mathrm{Au}\left(\Phi_{\mathrm{Au}}=5.40 \mathrm{eV}\right)^{12}$ and $\operatorname{Pt}\left(\Phi_{\mathrm{Pt}}=5.70 \mathrm{eV}\right)^{12}$ were chosen as the high work function metals, relative to the electron affinity of $\mathrm{MoS}_{2}$ $(\chi=4.0 \mathrm{eV}){ }^{6,13} \mathrm{~A}$ band diagram illustration of all the aforementioned metals relative to $\mathrm{MoS}_{2}$, prior to contact is shown in Fig. 3(a). Figure 3(b) shows the current-voltage (I-V) characteristics acquired for the different metal/MoS $\mathrm{M}_{2}$ contacts. It can be seen that $\mathrm{Al} / \mathrm{MoS}_{2}$ interface results in the highest current $(\sim 90 \mu \mathrm{A})$, while the $\mathrm{Pt} / \mathrm{MoS}_{2}$ interface shows the least current $(\sim 10 \mu \mathrm{A})$, under identical bias conditions.

When the metal and the $\mathrm{MoS}_{2}$ are brought in a contact, the bands of $\mathrm{MoS}_{2}$ bend such that the Fermi levels equilibrate. Figure 4(a) schematically illustrates the respective electronic band alignment of the metals for the case of $\Phi_{M}>\chi$ (in our case, the $\Phi_{M}$ of Al, W, Au and Pt all are larger than $\chi$ ), before the metal and $\mathrm{MoS}_{2}$ and brought into contact. On making contact, the conduction and valence bands of the $\mathrm{MoS}_{2}$ bend in order to achieve equilibrium between the metal Fermi level and the $\mathrm{MoS}_{2}$ chemical potential $(4.36 \mathrm{eV}) .{ }^{14}$ The degree and 
the direction of band bending depend on the work function of the metal relative to the Fermi level of the semiconductor $\left(\mathrm{MoS}_{2}\right.$ in this case). With $\mathrm{MoS}_{2}$ as the n-type semiconductor and for the case where the metal work function is larger than the electron affinity of $\mathrm{MoS}_{2}$, the depiction shown in Fig. 4(b) arises after the metal and semiconductor are brought into contact. In this case, the electrons diffuse from the semiconductor to the lower energy states of the metal in order to achieve a constant Fermi level throughout the system in thermal equilibrium. This results in the upward band bending within $\mathrm{MoS}_{2}$ at the interface. Consequently, the electrons in the metal see a potential barrier against their migration into the semiconductor. This barrier is known as the Schottky barrier $\left(\Phi_{\mathrm{B}}\right)$ which can be estimated by the Schottky-Mott rule $\left(\Phi_{\mathrm{B}}=\Phi_{\mathrm{M}}-\chi\right)$, where $\chi$ is the electron affinity of the semiconductor). A higher $\Phi_{M}$ thus results in a higher $\Phi_{\mathrm{B}}$. Hence, a larger forward bias is required in order to overcome this Schottky barrier.

This is confirmed from the $I-V$ characteristics (Fig. 3(b)), in which it is seen that the current is progressively higher for lower work function metals such as $\mathrm{Al}$ and $\mathrm{W}$ under identical bias conditions. Table I summarises the experimental results obtained using different metal contacts with varying work functions, relative to the electron affinity of $\mathrm{MoS}_{2}$. This shows that Al (the lowest work function metal) results in the highest bias current, indicating its superior room temperature charge injection capabilities for an interface with $\mathrm{MoS}_{2}$, compared to commonly used contact metals such as $\mathrm{Au}$ and Pt. The estimated Schottky barrier heights using the Schottky-Mott relationship are shown in Table I. We also calculated the Schottky barrier heights from the $I V$ curves. The calculated $\Phi_{\mathrm{B}}$ for $\mathrm{Al}$ $(0.55 \mathrm{eV})$ corresponds well to the theoretically estimated value. However, an accurate calculation for the other metals could not be obtained, as their relatively large $\Phi_{\mathrm{B}}$ required the application of higher bias voltages, causing the junction to break down. 
In summary, we investigated the properties of the metal/2D $\mathrm{MoS}_{2}$ contacts using metals of different work functions and demonstrated that for the chosen metals that have the work functions between 4.5 to $5.7 \mathrm{eV}$, always Schottky behaviours were observed. While, there are reports in literature that have claimed the $\mathrm{Au} / 2 \mathrm{D} \mathrm{MoS}$ contact to be Ohmic, our study clearly shows the formation of a barrier height and therefore Schottky behaviour for all of the metals under investigation. This study showed that the choice of the metal contacts is crucial in adjusting the barrier heights at their interface with $2 \mathrm{D} \mathrm{MoS}_{2}$, which is fundamental knowledge in designing electronic and optoelectronic devices based on 2D MoS 2 .

\section{ACKNOWLEDGMENTS}

The authors acknowledge support from the Australian Research Council (ARC) with partial project support through Discovery Project DP130100062; Australian Post-Doctoral Fellowships through Discovery Projects DP1092717 (MB) and DP110100262 (SS); and equipment funding through the Linkage, Infrastructure, Equipment, and Facilities Grant LE100100215 (SS and KKZ). SB acknowledges a publication grant from the School of Graduate Research at RMIT University.

\section{NOTES}

a) Corresponding authors. E-mail: madhu.bhaskaran@rmit.edu.au, kourosh.kalantar@ rmit.edu.au and $\underline{\text { sumeet.walia@rmit.edu.au }}$ 


\section{REFERENCES}

1. S. Balendhran, S. Walia, H. Nili, J. Z. Ou, S. Zhuiykov, R. B. Kaner, S. Sriram, M. Bhaskaran, and K. Kalantar-zadeh, Adv. Funct. Mater. 23, 3952 (2013).

2. Q. H. Wang, K. Kalantar-Zadeh, A. Kis, J. N. Coleman, and M. S. Strano, Nat. Nanotechnol. 7, 699 (2012).

3. D. J Late, B. Liu, H.S.S. Ramakrishna Matte, V. P. Dravid, and CNR Rao, ACS Nano 6, 5635 (2012).

4. B. Radisavljevic, A. Radenovic, J. Brivio, V. Giacometti, and A Kis, Nat. Nanotechnol. 6, $147(2011)$.

5. H. Wang, L. Yu, Y-H. Lee, Y. Shi, A. Hsu, M. L. Chin, L-J. Li, M. Dubey, J.Kong, and T. Palacios, Nano Lett. 12, 4674 (2012); Z. Yin, H. Li, H. Li, L. Jiang, Y. Shi, Y. Sun, G. Lu, Q. Zhang, X. Chen, and H. Zhang, ACS Nano 6, 74 (2011); G. Eda, H. Yamaguchi, D. Voiry, T. Fujita, M. Chen, and M. Chhowalla, Nano Lett. 11, 5111 (2011).

6. S. Das, H-Y. Chen, A. V. Penumatcha, and J. Appenzeller, Nano Lett. 13, 100 (2012).

7. Y. Yao, L. Tolentino, Z. Yang, X. Song, W. Zhang, Y. Chen, and C-P. Wong, Adv. Funct. Mater. 23, 3577 (2013).

8. See supplementary material at [URL to be inserted by AIP] for experimental details outlining the materials and methods used in this work.

9. Y. Wang, J. Z. Ou, S. Balendhran, A. F. Chrimes, M. Mortazavi, D. D. Yao, M. R. Field, K. Latham, V. Bansal, J. R. Friend, S. Zhuiykov, N.V. Medhekar, M. S. Strano, and K. Kalantarzadeh, ACS Nano, DOI: 10.1021/nn4041987 (2013).

10. S. Balendhran, J. Z. Ou, M. Bhaskaran, S. Sriram, S. Ippolito, Z. Vasic, E. Kats, S. Bhargava, S. Zhuiykov, and K. Kalantar-Zadeh, Nanoscale 4, 461 (2012).

11. D. J. Late, B. Liu, H. S. S. Ramakrishna Matte, C. N. R. Rao, and V. P. Dravid, Adv. Funct. Mater. 22, 1894 (2012).

12. H. Lomholt Skriver and N. M. Rosengaard, Phys. Rev. B. 46, 7157 (1992). 
13. K. Lee, H-Y. Kim, M. Lotya, J. N. Coleman, G-T. Kim, and G. S. Duesberg, Adv. Mater. 23, $4178(2011)$.

14. J-M. Yun, Y-J. Noh, J-S. Yeo, Y-J. Go, S-In Na, H-G. Jeong, J. Kim, S. Lee, S-S. Kim, and H. Y. Koo, J. Mater. Chem. C. 1, 3777 (2013).

TABLE I: Performance summary of different metal/2D $\mathrm{MoS}_{2}$ contacts

\begin{tabular}{cccc}
\hline \hline Metal & $\begin{array}{c}\text { Work function, } \\
\boldsymbol{\Phi}_{\mathbf{M}}(\mathbf{e V})\end{array}$ & $\begin{array}{c}\text { Schottky barrier height, } \\
\boldsymbol{\Phi}_{\mathbf{B}}=\boldsymbol{\Phi}_{\mathbf{M}}-\chi(\mathbf{e V})\end{array}$ & $\begin{array}{c}\text { Current at }+2 \mathbf{2 V} \text { bias } \\
(\boldsymbol{\mu A})\end{array}$ \\
$\mathrm{Al}$ & 4.5 & 0.5 & 91.9 \\
$\mathrm{~W}$ & 5.1 & 1.1 & 38.7 \\
$\mathrm{Au}$ & 5.4 & 1.4 & 22.5 \\
$\mathrm{Pt}$ & 5.7 & 1.7 & 17.3 \\
\hline \hline
\end{tabular}

\section{FIGURE CAPTIONS}

FIG. 1. (a) AFM image of typical exfoliated $\mathrm{MoS}_{2}$ nanoflakes with its corresponding crosssectional thickness profile. (b) DLS analysis of the lateral size distribution of the $\mathrm{MoS}_{2}$ nanoflakes.

FIG. 2. (a) Raman spectra comparison of bulk $\mathrm{MoS}_{2}$ and a nanostructured 2D MoS 2 thin film. (b) X-ray diffractograms comparing bulk $\mathrm{MoS}_{2}$ powder, a nanostructured thin film made of 2D $\mathrm{MoS}_{2}$ nanoflakes, with the FTO substrate spectrum provided for reference ${ }^{*}$ denotes the peaks corresponding to the FTO substrate). (c) Transmission electron micrograph of a liquid exfoliated 2D MoS $\mathrm{M}_{2}$ nanoflake with the inset corresponding to its high resolution transmission electron micrograph.

FIG. 3. (a) Band diagram illustrations of a metal and $\mathrm{MoS}_{2} \cdot \chi$ is the $\mathrm{MoS}_{2}$ electron affinity, $E_{\mathrm{F}}$ is the Fermi level of $\mathrm{MoS}_{2}, E_{\mathrm{VAC}}$ is the reference vacuum level, $E_{\mathrm{V}}$ and $E_{\mathrm{C}}$ are the valence and 
the conduction band levels respectively. (b) $I-V$ characteristics for different metal/MoS contacts under identical bias conditions.

FIG. 4. Band alignments of a metal and $\mathrm{MoS}_{2}$ for $\Phi_{M}>\chi$ (a) before establishing a contact and (b) their corresponding band bending after establishing a contact. 


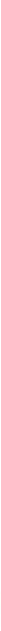

Raman Shift $\left(\mathrm{cm}^{-1}\right)$

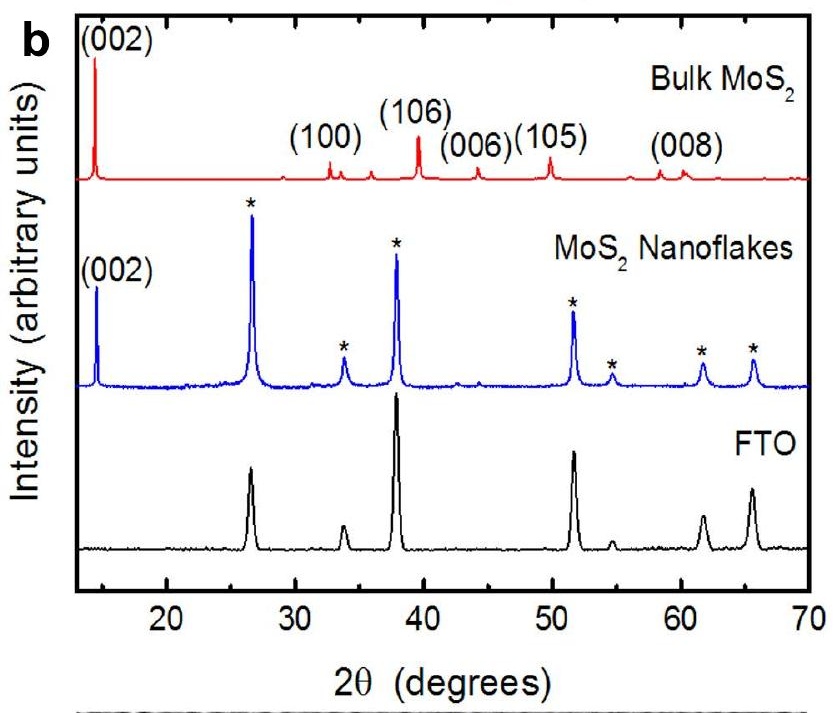

C

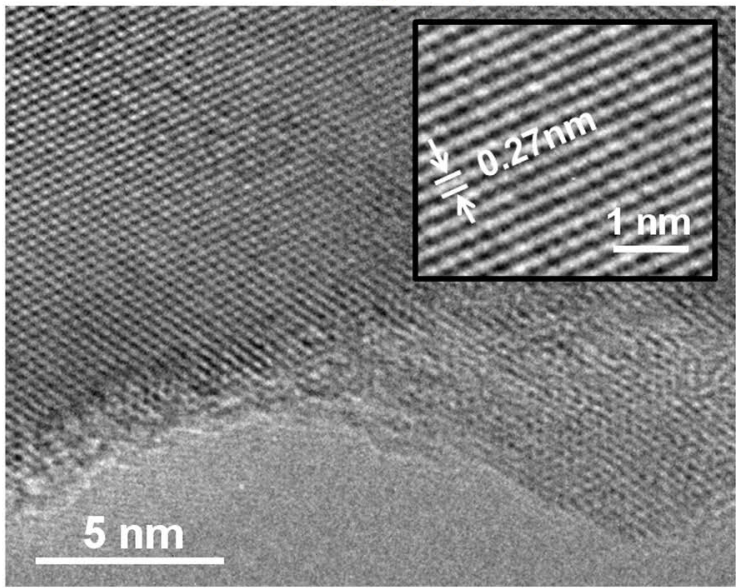


